\title{
CAPTURA DE DIÓXIDO DE CARBONO UTILIZANDO ÓXIDOS A BASE DE CÁLCIO
}

\author{
L. V. BISINOTI ${ }^{1}$, J. A. SILVA ${ }^{1}$ e J. B. O. SANTOS ${ }^{1}$ \\ ${ }^{1}$ Universidade Federal de São Carlos, Departamento de Engenharia Química \\ E-mail para contato: joaobatista@ufscar.br
}

\begin{abstract}
RESUMO - A captura de dióxido de carbono $\left(\mathrm{CO}_{2}\right)$ foi realizada por ciclos de carbonatação de materiais contendo óxido de cálcio. Entre cada ciclo de carbonatação foi realizada a decomposição do carbonato de cálcio formado com formação de $\mathrm{CO}_{2}$ com elevada pureza. A reação de carbonatação foi conduzida a $700{ }^{\circ} \mathrm{C}, 1 \mathrm{~atm}$ e corrente de $\mathrm{CO}_{2}$ puro, enquanto a decomposição foi realizada a $800{ }^{\circ} \mathrm{C}$ em corrente de nitrogênio. Os materiais estudados foram óxido de cálcio, preparado pelo método sol-gel, e uma mistura contendo $50 \%$ em massa de óxido de cálcio e $50 \%$ em massa de alumina. Os materiais foram caracterizados por difração de raios x e adsorção de nitrogênio. A captura de $\mathrm{CO} 2$ no óxido de cálcio puro foi de aproximadamente $0,70 \mathrm{~g}$ de $\mathrm{CO} 2$ por grama de óxido de cálcio, enquanto sobre a mistura óxido de cálcio-alumina foi de $0,10 \mathrm{~g}$ de $\mathrm{CO}_{2}$ por $\mathrm{g}$ do material. O material contendo alumina foi mais resistente durante os ciclos de reação do que o óxido de cálcio puro.
\end{abstract}

\section{INTRODUÇÃO}

Os gases do efeito estufa, em inglês, GHG (Greenhouse Gases), existem naturalmente na atmosfera. Entretanto, com o advento da revolução industrial e o aumento no consumo de combustíveis fósseis ocorreu um crescimento exagerado na concentração desses gases na atmosfera, considerados os grandes responsáveis pelo aumento da temperatura terrestre vivenciado nos últimos anos, fenômeno conhecido como aquecimento global (Qin et al., 2015).

O dióxido de carbono $\left(\mathrm{CO}_{2}\right)$ é o principal GHG e, na história recente, apresentou um aumento de emissões sem precedentes. Por isso, fontes estacionárias que utilizam combustão de combustíveis fósseis, como usinas termoelétricas e indústrias de aço e cimento, são consideradas as maiores responsáveis pelo aquecimento global.

A captura e armazenamento de $\mathrm{CO}_{2}$ é uma alternativa promissora para a redução das emissões de GHG, por meio do abatimento do $\mathrm{CO}_{2}$ emitido por fontes estacionárias. Atualmente, a tecnologia mais empregada para este fim é a de absorção por aminas; porém, os altos custos e os problemas relacionados à sua degradação e perda vêm direcionando diversas pesquisas para o desenvolvimento de materiais sólidos para captura de $\mathrm{CO}_{2}$ ( $\mathrm{Lu}$ et al., 2014).

De maneira geral, os gases provenientes da queima de carvão ou gás natural possuem em média uma concentração de 10 a $20 \%$ de $\mathrm{CO}_{2}$ e a captura deste gás pode ser feita pela reação entre o óxido de cálcio $(\mathrm{CaO})$ e o $\mathrm{CO}_{2}$. O processo é conhecido como Ca-looping 
reaction, ou seja, o $\mathrm{CO}_{2}$ reage com o $\mathrm{CaO}$ para formar carbonato de cálcio e, em seguida, o carbonato pode passar por um reator de decomposição, gerando o $\mathrm{CO}_{2}$ e regenerando o $\mathrm{CaO}$, o qual pode ser utilizado em um novo processo. Em outras palavras, reações gás-sólido ocorrem de forma cíclica para capturar o $\mathrm{CO}_{2}$.

O baixo custo de $\mathrm{CaO}$, a elevada capacidade de captura de $\mathrm{CO}_{2}$ (cerca de 0,786 g de $\mathrm{CO}_{2}$ por $\mathrm{g}$ de óxido) e a cinética rápida das reações de calcinação e carbonatação são as vantagens encontradas no processo. No entanto, a grande desvantagem do $\mathrm{CaO}$ é a perda da área superficial pela exposição do sólido aos ciclos de carbonatação e decomposição, os quais são realizados em altas temperaturas. A perda da área superficial deve-se ao fenômeno de sinterização do material (Lu et al., 2014; Sun et al., 2007).

Três possibilidades para melhorar a performance de captura dos óxidos a base de cálcio submetidos a diversos ciclos de calcinação-carbonatação são: (1) a adição de suportes inertes, (2) técnicas avançadas de preparação, como a síntese pelo método sol-gel (Xu et al., 2016) e (3) a modificação da estabilidade e estrutura dos materiais.

Neste trabalho, dois materiais a base de cálcio foram preparados e utilizados na captura de $\mathrm{CO}_{2}$. O objetivo principal do trabalho foi verificar a estabilidade dos sólidos nas reações de carbonatação e decomposição. Os materiais utilizados foram: (i) $\mathrm{CaO}$ preparado pelo método sol-gel e (ii) $\mathrm{CaO}$ impregnado com $\mathrm{Al}_{2} \mathrm{O}_{3}$.

\section{PARTE EXPERIMENTAL}

\subsection{Preparação dos Materiais}

Os dois materiais a base de cálcio, $\mathrm{CaO}$ puro e uma mistura entre $\mathrm{CaO}$ e $\mathrm{Al}_{2} \mathrm{O}_{3}$, foram preparados utilizando reagentes da empresa Sigma-Aldrich. A descrição da preparação de cada material é apresentada a seguir.

Sol-gel: Foram empregados os procedimentos propostos por Lee et al. (2015) e Xu et al. (2016). O precursor utilizado para síntese foi o nitrato de cálcio tetrahidratado e foi usado ácido cítrico como agente quelante. Os íons metálicos foram agitados e dissolvidos em uma proporção de 1:3 para o ácido cítrico e de 1:125 para água destilada. A solução formada foi agitada e aquecida a $80^{\circ} \mathrm{C}$ até que o gel fosse formado. Após envelhecimento por $12 \mathrm{~h}$, o gel foi seco a $100^{\circ} \mathrm{C}$ por $12 \mathrm{~h}$ e, em seguida, calcinado a $800^{\circ} \mathrm{C}$ durante $4 \mathrm{~h}$.

$\underline{\mathrm{CaO}-\mathrm{Al}_{2}} \underline{\mathrm{O}}_{3}$ : Alumina em pellets foi macerada e peneirada em 100 Ty $(150 \mu \mathrm{m})$. Foi utilizada a proporção de $50 \%$ de $\mathrm{Al}_{2} \mathrm{O}_{3}$ para $50 \%$ de $\mathrm{CaO}$. Foi adicionado $50 \mathrm{~mL}$ de etanol ao nitrato de cálcio utilizado como precursor e a solução foi agitada até completa diluição, para que, então, a alumina fosse adicionada. Após a adição da alumina, a solução foi agitada por 2 $\mathrm{h}$ a temperatura ambiente. Então, a solução foi levada para o rotaevaporador em banho de 60 ${ }^{\circ} \mathrm{C}$ e $80 \mathrm{rpm}$ até a secagem total do material. O material foi secado a $80{ }^{\circ} \mathrm{C}$ por $12 \mathrm{~h} \mathrm{e}$ calcinado a $800^{\circ} \mathrm{C}$ por $4 \mathrm{~h}$.

\subsection{Caracterização dos materiais}


Para caracterização dos materiais foi realizada a análise por difração de raios-x (XRD) das amostras utilizando o difratômetro Miniflex, com radiação do $\mathrm{Cu}$. $\mathrm{O}$ equipamento foi operado a $40 \mathrm{kV}$ e $40 \mathrm{~A}$, com varredura de $2 \theta=10$ a 90.

As isotermas de adsorção de $\mathrm{N}_{2}$ foram feitas por meio de um equipamento automatizado de análise de adsorção de gases. A área superficial específica foi determinada pela aplicação do método Brunauer-Emmett-Teller (BET) e o volume total de poros pelo método de BarretJoyner-Halenda (BJH).

\subsection{Captura de $\mathrm{CO}_{2}$}

As taxas de captura foram obtidas por análise termogravimétrica (TGA) utilizando uma termobalança da marca Shimadzu. Inicialmente foi feito o aquecimento de aproximadamente $10 \mathrm{mg}$ de amostra até $800{ }^{\circ} \mathrm{C}$ em atmosfera de nitrogênio para eliminação de possíveis impurezas e de $\mathrm{CaCO}_{3}$. A temperatura de carbonatação adotada foi de $700{ }^{\circ} \mathrm{C}$ e a temperatura de calcinação de $800^{\circ} \mathrm{C}$. Foram realizados 6 ciclos completos para cada amostra e, entre as mudanças de atmosfera de $\mathrm{N}_{2}$ para $\mathrm{CO}_{2}$ (e vice-versa), as amostras foram submetidas a uma atmosfera de $\mathrm{N}_{2}$ por 5 minutos.

A capacidade de captura de $\mathrm{CO}_{2}$ e o rendimento da reação foram calculados pelas Equações 1 e 2, respectivamente:

$$
\begin{aligned}
& \mathrm{C}_{\mathrm{N}}=\left(\mathrm{m}_{\mathrm{N}}-\mathrm{m}_{0}\right) / \mathrm{m}_{0} \\
& \mathrm{X}_{\mathrm{N}}=\frac{\left(\mathrm{m}_{\mathrm{N}}-\mathrm{m}_{0}\right)}{\text { b. } \mathrm{m}_{0}} \frac{\mathrm{M}_{\mathrm{CaO}}}{\mathrm{M}_{\mathrm{CO}_{2}}}
\end{aligned}
$$

Em que $\mathrm{C}_{\mathrm{N}}$ e $\mathrm{X}_{\mathrm{N}}$ são a captura de $\mathrm{CO}_{2}$ e a conversão de $\mathrm{CaO}$ no enésimo ciclo, $\mathrm{m}_{\mathrm{N}}$ é a massa final após a enésima carbonatação, $\mathrm{m}_{0}$ é a massa de material após a calcinação inicial, $\mathrm{b}$ é o porcentual de $\mathrm{CaO}$ no material, $\mathrm{M}_{\mathrm{CaO}}$ e $\mathrm{M}_{\mathrm{CO} 2}$ as massas molares do $\mathrm{CaO}$ e do $\mathrm{CO}_{2}$.

\section{RESULTADOS E DISCUSSÕES}

As Figuras 1 e 2 apresentam a quantidade de $\mathrm{CO}_{2}$ capturada em cada ciclo de carbonatação para o $\mathrm{CaO}$ e $\mathrm{CaO}-\mathrm{Al}_{2} \mathrm{O}_{3}$, respectivamente. Para o material sintetizado via solgel observou-se um aumento na capacidade de captura até o terceiro ciclo e, a partir deste ponto, a capacidade de captura de $\mathrm{CO}_{2}$ começou a diminuir. Os resultados encontrados na literatura (Lee et al., 2015; $\mathrm{Xu}$ et al., 2016) indicam que a captura de $\mathrm{CO}_{2}$ utilizando $\mathrm{CaO}$ diminuiu com os ciclos, o que está de acordo com os resultados apresentados neste trabalho. Para o material impregnado com alumina observa-se um um crescimento gradual na capacidade de captura até o terceiro ciclo, sendo que a capacidade de captura de $\mathrm{CO} 2$ permanece praticamente constante nos demais ciclos. $\mathrm{O}$ aumento da capacidade de captura em ambas as amostras nos primeiros ciclos pode ser atribuido a instabilidade do sistema reacional no início do processo.

A queda da capacidade de captura observado no $\mathrm{CaO}$ preparado pelo método sol-gel pode ser atribuído a sinterização do material. De fato, vários autores (Lee et al., 2015; Xu et al., 2016) tem reportado a perda de atividade de materiais a base de cálcio devido a 
sinterização. O material contendo alumina se mostrou estável durante os ciclos, indicando que a alumina inibiu o processo de sinterização do material.

Figura 1 - Capacidade de captura de $\mathrm{CO}_{2}$ do $\mathrm{CaO}$ preparado pelo método sol gel em função do número de ciclos.

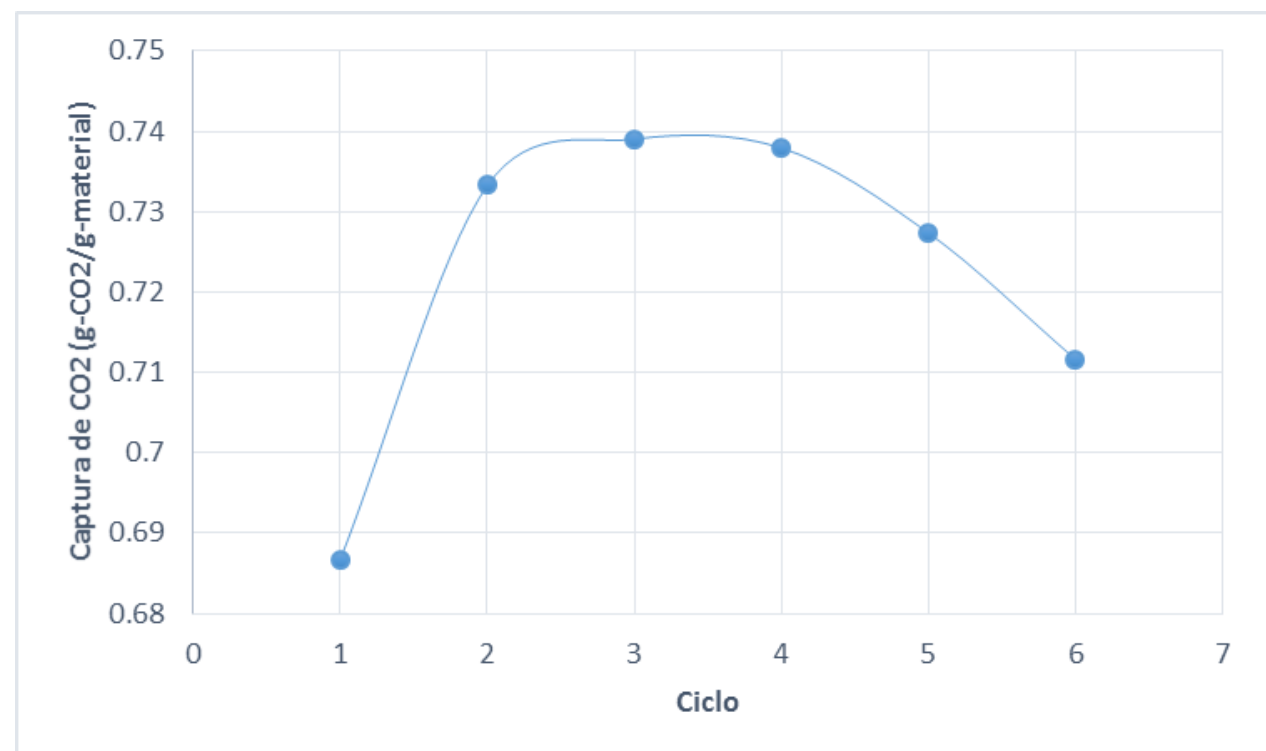

Figura 2 - Capacidade de captura de $\mathrm{CO}_{2}$ do $\mathrm{CaO}$ impregnado com $\mathrm{Al}_{2} \mathrm{O}_{3}$ em função do número de ciclos.

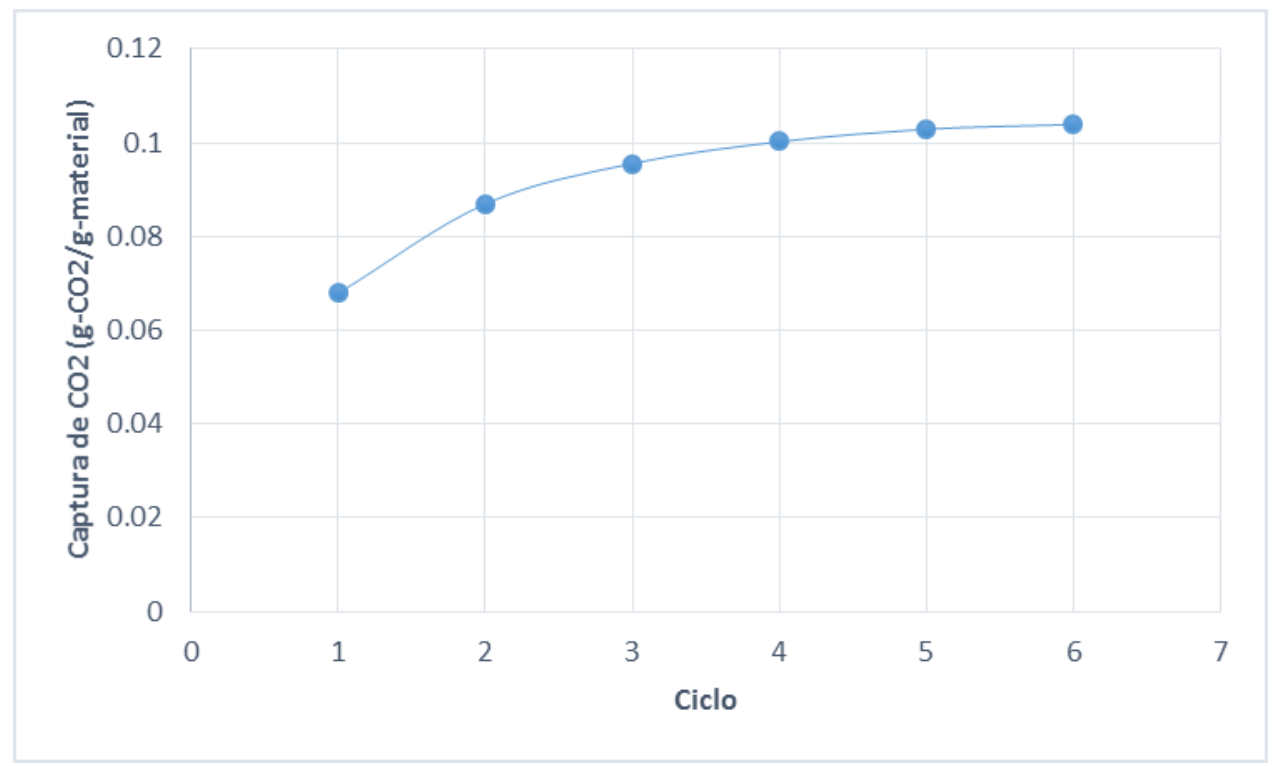

A capacidade de captura de $\mathrm{CO}_{2}$ foi muito maior para o $\mathrm{CaO}$ puro em comparação ao material contendo alumina. A conversão no último ciclo foi de cerca de $90 \%$ para o $\mathrm{CaO}$ e de $25 \%$ para a amostra contendo alumina. Este fato pode ser explicado pela perda de área superficial do $\mathrm{CaO}$ e do volume de poros do material após a adição da alumina. Entretanto, a observação experimental da redução na área do $\mathrm{CaO}$ é dificil, pois a área da alumina é muito 
maior e acaba interferindo na análise. As áreas $\mathrm{BET}$ do $\mathrm{CaO}$ e do $\mathrm{CaO}-\mathrm{Al}_{2} \mathrm{O}_{3}$ foram 22 e 80 $\mathrm{m}^{2} / \mathrm{g}$, indicando uma redução na área de $\mathrm{CaO}-\mathrm{Al}_{2} \mathrm{O}_{3}$ em relação a alumina pura $\left(100 \mathrm{~m}^{2} / \mathrm{g}\right)$.

As caracterizações de XRD para as amostras $\mathrm{CaO}$, preparado pelo método sol-gel, e $\mathrm{CaO}-\mathrm{Al}_{2} \mathrm{O}_{3}$ são apresentadas nas Figuras 3 e 4, respectivamente. Os picos de difração indicam uma mistura contendo óxido de cálcio e carbonato de cálcio para a amostra $\mathrm{CaO}$. $\mathrm{Na}$ amostra contendo alumina, além da formação do óxido de calcio e de carbonato, ocorre a formação de novos picos de difração, indicando a formação de um possível aluminato de cálcio. A formação de carbonato de cálcio nas amostras calcinadas e expostas ao ar também foi observado nas análises termogravimétricas.

Figura 3 - Difratograma de raios x para a amostra de $\mathrm{CaO}$ preparada pelo método sol-gel.

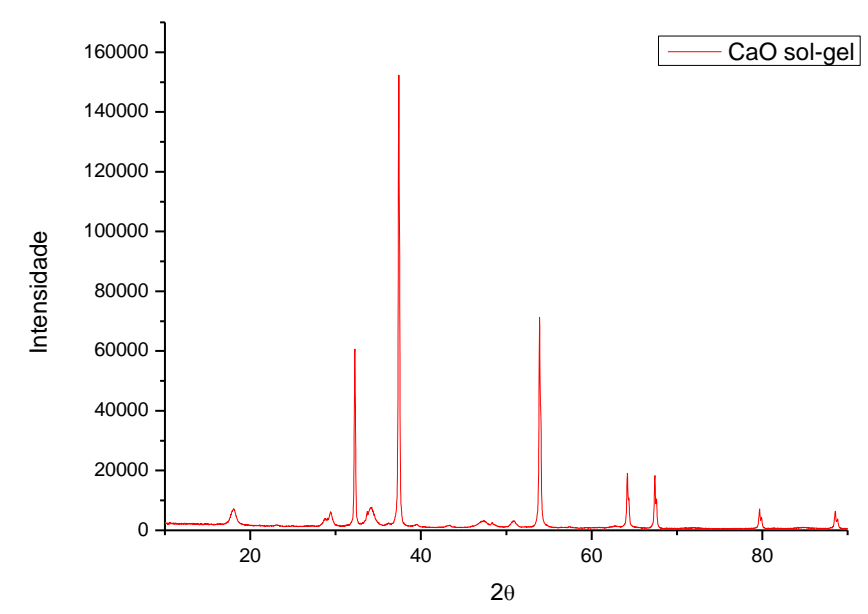

Figura 4 - Difratograma de raios x para a amostra contendo $\mathrm{CaO}-\mathrm{Al}_{2} \mathrm{O}_{3}$.

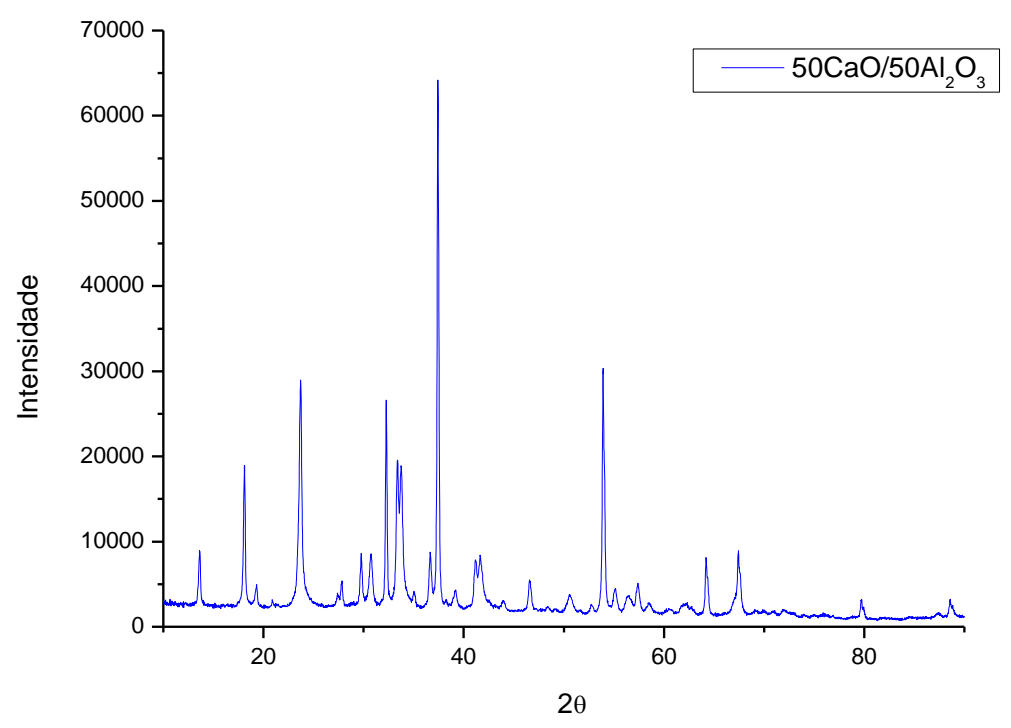




\section{CONCLUSÃO}

A carbonatação de $\mathrm{CaO}$ ocorre com alta eficiência, mas a capacidade de captura de $\mathrm{CO} 2$ diminuiu com os ciclos devido a sinterização da amostra. A adição de alumina ao $\mathrm{CaO}$ inibe a sinterização da amostra durante os ciclos de carbonatação e decomposição; entretanto, a eficiência de captura de $\mathrm{CO}_{2}$ diminui.

A difração de raios $\mathrm{x}$ indicou a formação de $\mathrm{CaO}$ e carbonato de cálcio na amostra preparada pelo método sol-gel. Já a amostra contendo alumina foi constituída de $\mathrm{CaO}$, carbonato de cálcio e aluminato de cálcio.

\section{REFERÊNCIAS}

LEE, Z.; ICHIKAWA, S.; LEE, K.; MOHAMED, A. The role of nickel oxide additive in lowering the carbon dioxide sorption temperature of CaO. J. Energy Chem., v.24, p. 225-231, 2015.

LU, A.; HAO, G.; ZHANG, X. Porous Materials for Carbon Dioxide Capture. Green Chemistry and Sustainable Technology, p. 15-77, 2014.

SUN, P.; GRACE, J. R.; LIM, C. J.; ANTHONY, E. J. The effect of CaO sintering on cyclic CO2 capture in energy systems. Aiche Journal, v. 53, p. 2432-42, 2007.

QIN, C.; YIN, J.; FENG, B.; RAN, J.; ZHANG, L.; MANOVIC, V. Modelling of the calcination behaviour of a uniformly-distributed $\mathrm{CuO} / \mathrm{CaCO} 3$ particle in $\mathrm{Ca}-\mathrm{Cu}$ chemical looping. Applied Energy, v. 164, p. 400-410, 2016.

XU, Y.; LUO, C.; ZHENG, Y.; HAORAN, D.; QIYAO, W.; XIAOSHAN, L.; LIQI, Z. Characteristics of CaO-base high temperature $\mathrm{CO}_{2}$ sorbents derived from a sol-gel process with different suports. RSC Adv., v.6, p. 79285-79296, 2016. 